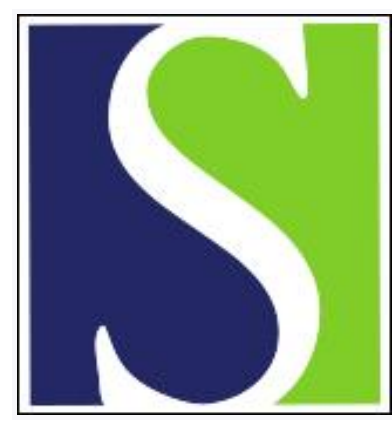

Scand J Work Environ Health 2009;35(3):212-221

https://doi.org/10.5271/sjweh.1326

Published online: 08 May 2009, Issue date: 00 May 2009

The occupational exposure of dermatology nurses to polycyclic aromatic hydrocarbons - evaluating the effectiveness of better skin protection

by Scheepers PTJ, van Houtum J, Anzion RBM, Champmartin C, Hertsenberg S, Bos RP, van der Valk P

Affiliation: Department of Epidemiology, Biostatistics and HTA (133 EBH), Radboud University Nijmegen Medical Centre, PO Box 9101, 6500 HB Nijmegen, the Netherlands. p.scheepers@ebh.umcn.nl

Key terms: $\mathrm{BaP}$; benzo[a]pyrene; biological monitoring; coal tar; dermatology nurse; glove; nurse; occupational exposure; PAH; polycyclic aromatic hydrocarbon; PYR; pyrene; skin absorption; skin protection; urinary excretion

This article in PubMed: www.ncbi.nlm.nih.gov/pubmed/19430709 


\title{
The occupational exposure of dermatology nurses to polycyclic aromatic hydrocarbons - evaluating the effectiveness of better skin protection
}

\author{
By Paul TJ Scheepers, PhD, ${ }^{1}$ Jeanette van Houtum, MD, ${ }^{2}$ Rob BM Anzion, BSc, ${ }^{1}$ Catherine Champmartin, \\ PhD, ${ }^{3}$ Selma Hertsenberg, MSc, ${ }^{1}$ Rob P Bos, PhD, ${ }^{4}$ Pieter van der Valk, MD ${ }^{2}$
}

\begin{abstract}
Scheepers PTJ, van Houtum J, Anzion RBM, Champmartin C, Hertsenberg S, Bos RP, van der Valk P. The occupational exposure of dermatology nurses to polycyclic aromatic hydrocarbons - evaluating the effectiveness of better skin protection. Scand J Work Environ Health 2009;35(3):212-221.
\end{abstract}

\begin{abstract}
Objectives We studied the uptake of polycyclic aromatic hydrocarbons (PAH) in nurses who apply ointments containing coal tar to patients and investigated the effectiveness of skin protection methods.

Methods We determined gas-phase PAH on XAD-2 and particle-associated PAH on filters. We also used pads to determine PAH on the skin. Pyrene and benzo(a)pyrene were analyzed by gas chromatography/mass spectrometry and gas chromatography/tandem mass spectrometry; their respective urinary metabolites 1hydroxypyrene and 3-hydroxybenzo(a)pyrene were analyzed using high performance liquid chromatography with fluorescence detection.
\end{abstract}

Results We ruled out the inhalation of airborne pyrene and benzo(a)pyrene as the sources of PAH expo-
sure. However, substantial amounts of pyrene and benzo(a)pyrene were observed on the hands of the nurses
(median 33.0 and $16.4 \mathrm{ng} / \mathrm{cm}^{2}$, respectively). Excretion of urinary 1 -hydroxypyrene indicated an increased
uptake of pyrene in 8 out of 12 nurses. We asked 35 nurses to perform a treatment with gloves followed by a
second treatment without gloves. The use of gloves changed the excretion of 1 -hydroxypyrene by $-0.58 \mu \mathrm{mol}$
(range $-5.1-1.0 \mu \mathrm{mol})$, corresponding to a median reduction of $51.5 \%(\mathrm{P}<0.001)$. Based on this finding, a new
protocol was adopted, involving the permanent use of vinyl gloves and Tyvek sleeves. The effectiveness of this
protocol was tested against pre-existing work practices and showed a $97 \%$ reduction in skin contamination with
pyrene and benzo(a)pyrene, and a lowering in urinary excretion of 1 -hydroxypyrene of $57 \%$.

Conclusion Protecting the skin more stringently reduced pyrene and benzo(a)pyrene contamination of the hands, and lowered urinary excretion of 1-hydroxypyrene.

Key terms coal tar; benzo[a]pyrene (BaP); pyrene (PYR); skin absorption; urinary excretion; biological monitoring; gloves.

Coal tar has been used in dermatology for many decades. Although evidence from controlled trials is scarce, dermatologists consider (short-term) coal tar treatment of psoriasis and atopic dermatitis to be relatively safe and efficacious (1-3). Coal tar is a complex mixture of organic compounds that is not completely chemically characterized. It contains polycyclic aromatic hydrocarbons (PAH) including some human carcinogens (4). Coal tar is a second choice topical therapy. It is used to support first line treatment with dermatocorticosteroids or topical calcineurin inhibitors (in case of eczema), and dermatocorticosteroids and/or vitamin D derivatives (in case of psoriasis). Dutch dermatologists mainly use coal tar in the treatment of eczema $(72 \%$ versus $48 \%$ in Belgian Flanders), whereas in Flanders, coal tar is mainly prescribed for psoriasis $(60 \%$ versus $41 \%$ in The Netherlands) (5-6).

Patients treated with coal tar ointment (CTO) excrete mutagenic metabolites in urine (7). A component of CTO, pyrene is metabolized to 1-hydroxypyrene (1-OHP) and has been observed in high concentrations in the urine of patients treated for psoriasis (8). Pyrene and

1 Research Laboratory Molecular Epidemiology, Department of Epidemiology, Biostatistics and Human Technology Assessment, Radboud University Nijmegen Medical Centre, PO Box 9101, 6500 HB Nijmegen, the Netherlands.

2 Department of Dermatology, Radboud University Nijmegen Medical Centre, PO Box 9101, 6500 HB Nijmegen, the Netherlands.

3 Institut National de Recherche et de Sécurité, Avenue de Bourgogne, 54501 Vandoeuvre Cedex, France.

4 Department of Pharmacology-Toxicology, Radboud University Nijmegen Medical Centre, PO Box 9101, 6500 HB Nijmegen, the Netherlands.

Correspondence to: Dr PTJ Scheepers, Department of Epidemiology, Biostatistics and HTA (133 EBH), Radboud University Nijmegen Medical Centre, PO Box 9101, 6500 HB Nijmegen, the Netherlands. [E-mail p.scheepers@ebh.umcn.nl] 
1-OHP, although not mutagenic, can be used as markers for total poorly volatile (particulate) PAH exposure and as indicators for systemic exposure to mutagenic PAH (9). Also benzo[a]pyrene $(\mathrm{BaP})$ and 3-hydroxybenzo(a)pyrene (3-OHBaP) have been proposed as markers for external and internal exposure to carcinogenic (high molecular weight) PAH, respectively (10).

Several studies have shown an increased risk of skin and internal cancer in subjects with long-term coal tar exposure (8-18). An increase in cancer risk following long-term occupational exposure, although not established for dermatology nurses, cannot be ruled out per se. Therefore, exposure to PAH in nurses applying tar products to patients' skin must be limited as much as possible. In our hospital, we advise our nursing personnel to wear gloves when applying tar. However, applying tar with gloves is not always convenient for the patient and the nurses are inclined, especially in the treatment of children, to ignore this advice.

We did not know to what extent wearing gloves protects against coal tar exposure. To investigate the possible uptake of $\mathrm{PAH}$ when administering $\mathrm{CTO}$, we studied the exposure of nurses involved in the topical application of CTO in the dermatology clinic. This paper summarizes our efforts to determine pyrene and $\mathrm{BaP}$ levels in the air around and on the skin of nurses, and the excretion of their respective metabolites 1-hydroxypyrene (1-OHP) and 3-hydroxybenzo[a]pyrene (3-OHBaP) in urine as biomarkers of internal exposure. This study was aimed at investigating a possible increased occupational uptake of $\mathrm{PAH}$ and finding out about the effectiveness of a more adequate and rigorous protocol of skin protection.

\section{Study population and methods}

\section{Subjects}

The nurses who volunteered to participate in the study signed a consent form stating they confirmed to have received information about the objectives of the study. The results of the urine analyses were given to the occupational physician, along with a list of the names of the workers and the personal codes. This was so that the results of the biological monitoring study could be added to the medical files and be available to the nurses on request.

The subjects were studied on the day that they treated one or more patients and during the following day(s). During the sampling process, the nurses were asked to refrain from consuming smoked, fried, grilled, or barbecued fish or meat in order to prevent the influence of diet on the pattern of urinary excretion of 1-OHP and 3-OHBaP (8-9, 19-20).

\section{Study design}

Four different studies were performed. In each study, the nurses were observed following the administration of one or more treatments of CTO per shift. A single treatment normally takes 10-30 minutes. The first study (A) was designed to investigate the contribution of inhalation and skin absorption as the two most obvious routes of uptake of PAH due to occupational exposures. During a subsequent study (B), we followed three nurses during a prolonged period of time and collected spot urine samples in order to study the relationship between work tasks and urinary excretion pattern of $\mathrm{PAH}$-metabolites. In a third study (C), we followed 35 nurses on two separate occasions involving CTO applications. This treatment was carried out on the same patient, so the amount of applied CTO, body parts treated, and the duration of the treatment were similar. On the first occasion, protective gloves were used and, during the second occasion, no gloves were used. By comparing these two situations, we were able to quantify how wearing gloves can reduce the skin absorption of PAH. In a fourth and last study (D), a new work procedure was tested for its effectiveness in protecting the nurses from the exposure of hands and forearms by using vinyl gloves (Flexam vinyl, supplied by Cardinal Health, Dublin, OH, USA) combined with Tyvek ${ }^{\circledR}$ narrow sleeves (model PS32LA, Du Pont Personal Protection, Amsterdam, The Netherlands). The effectiveness of this new work procedure was tested against results of urinary excretion of 1-OHP obtained in study A. An overview of the study components is presented in table 1.

\section{Questionnaires}

Using a questionnaire, we collected information on each nurse's occupational history and smoking habits, and uptake of PAH from the diet and exposure to sources of indoor and outdoor open fire, among others. This questionnaire also contained a number of questions generally describing working practices. A second questionnaire was completed on each day of urine collection. This questionnaire covered diet, active smoking, consumption of alcoholic beverages, use of medication, and some more specific questions about daily work practices (eg, the amount and type of CTO administered to the patient, the use of gloves and protective garments, and personal hygiene such as the time between CTO administration and washing of hands).

\section{Treatment with coal tar ointment}

Coal tar is applied either directly to lesions, in the case of limited disease, or diffusely if the lesions are disseminated. In the dermatology clinic, two different 
Table 1. Overview of study parameters. $(A M=$ arithmetic mean, $P A S=$ personal air sampling $)$

\begin{tabular}{|c|c|c|c|c|c|c|c|c|c|}
\hline \multirow[t]{2}{*}{ Study } & \multicolumn{3}{|c|}{$\mathrm{N}$} & \multirow{2}{*}{$\begin{array}{l}\text { Inhalation } \\
\text { exposure }\end{array}$} & \multirow{2}{*}{$\begin{array}{c}\text { Skin } \\
\text { exposure }\end{array}$} & \multirow[t]{2}{*}{ Type of skin protection } & \multirow{2}{*}{$\frac{\text { Treatments AM }}{\text { N Range }}$} & \multicolumn{2}{|c|}{ Urine collection } \\
\hline & Total & Vome & Men & & & & & Period & Type of sample \\
\hline A & $12^{\mathrm{a}}$ & 9 & 3 & PAS & Pads & With loose-fit polyethylene gloves & $1.21-2$ & 48 hours & Total void \\
\hline B & $3^{b}$ & 3 & 0 & . & . & With and without loose-fit polyethylene gloves & $7.06-8$ & 23 days & Spot sample \\
\hline $\mathrm{C}$ & $35^{b}$ & 30 & 5 & . & . & With and without loose-fit polyethylene gloves & $1.0 \quad .$. & 10 hours & Total void \\
\hline D & $16^{\mathrm{a}}$ & 12 & 4 & . & Pads & With tight-fit vinyl gloves and Tyvek® narrow sleeves & $2.42-4$ & 24 hours & Total void \\
\hline
\end{tabular}

a Three smokers.

${ }^{\mathrm{b}}$ All non-smokers.

types of CTO are applied in various formulations. Used in the treatment of chronic eczema, "dark" colored CTO contains $5 \%$ crude coal tar, $965 \mu \mathrm{g} / \mathrm{g}$ pyrene and $455 \mu \mathrm{g} / \mathrm{g} \mathrm{BaP}$ (the ratio of $\mathrm{BaP}$ to pyrene is 0.47 ). "Light" colored CTO, use to treat mild dermatitis and psoriasis, contains an alcoholic extract of crude coal $\operatorname{tar}(10 \%$ solution liquor carbonis) with a much lower PAH content $(3.0 \mu \mathrm{g} / \mathrm{g}$ pyrene and $0.13 \mu \mathrm{g} / \mathrm{g} \mathrm{BaP}$, with a $\mathrm{BaP}$ to pyrene ratio of 0.043$)$. The nurse noted the type of CTO used and determined the amount applied by weighing the container of CTO before and after each single treatment. From the total amount of CTO and the type of CTO used, the total amount of pyrene and $\mathrm{BaP}$ was calculated.

\section{Air samples}

Task-based measurements of volatile and particulate PAH were conducted only during the treatment of the patient (approximately 10-60 minutes per patient). Volatile PAH were collected on Amberlite XAD-2 (Sigma-Aldrich Chemie, Zwijndrecht, the Netherlands) placed in the breathing zone of each nurse. The cartridge was connected to an automated electronic personal air sampling pump (DuPont Alpha-1, Deha International, Huizen, the Netherlands) operated at a flow of $0.201 / \mathrm{min}$. Airborne particle-associated PAH were monitored using a Teflon filter (Type TE38, Schleicher and Schüll, Dassel, Germany) placed in an air sampling probe for the collection of inhalable particles (according to EN481). Air was drawn through this filter at a flow of $23.6 \mathrm{l} / \mathrm{min}$ using a medium volume air sampling pump (Environmental Monitoring Systems, Charleston, SC, USA).

\section{Skin pads}

Skin pads were homemade using polypropylene membrane filters embedded in self-adhesive Fixomull stretch elastic bandage (BSN Medical, Almere, the Netherlands); see Jongeneelen et al (21) for a more detailed description. These pads were attached to the skin on three standardized places on the dominant hand: (i) the lateral side of the index finger, (ii) the hand palm, and (iii) the lateral side of the wrist. The pads were attached just before the start of the treatment of the patient with CTO and removed again directly upon completion of the treatment. The pads were wrapped in aluminium foil and kept at $-20^{\circ} \mathrm{C}$ until analysis.

\section{Collection of urine samples}

Urine was collected in $250 \mathrm{ml}$ polyethylene (plastic) bottles with screw caps and a label registering the name and date of birth of the subject, and the date and time of the sample collection. Each subject received a sheet with instructions for urine collection. Urine was first collected in a measuring beaker $(500 \mathrm{ml})$ and the total volume of the void was written down on a registration form. An aliquot of the sample was transferred to the bottle and the residue was flushed away. Urine samples were kept in a cool and dark place, and taken to a central storage facility at the Dermatology department. Three different schemes for urine collection were used. In study $\mathrm{A}$, we collected all the urine samples on the same and consecutive day until 48 hours after the time of CTO application. In study B, spot urine samples were collected between 9:00-22.00 hours (post-shift). In study C, a pre-shift spot sample was collected as the first void after awakening. To reduce the workload for the volunteers in this study, urine samples were collected between 8.00-18.00 hours following the application of the CTO. In study D, we used the design of study A and modified only the period of urine collection to 24 instead of 48 hours since these samples were not analyzed for 3-OHBaP.

\section{Analysis from air filters and skin pads}

The polypropylene filters were punched from the skin pads and extracted in $10 \mathrm{ml}$ of dichloromethane (high performance liquid chromatography grade purchased from Lab-Scan, Gliwice, Poland). The filters were vortexed for 1 minute followed by 15 minutes in an ultrasonic bath. The Teflon air filters and XAD-2 solid sorbent cartridges were extracted following the same procedure. After removing the filters, the extracts 
were evaporated under a gentle stream of nitrogen at $25^{\circ} \mathrm{C}$. The residue was re-dissolved in toluene (Merck, Darmstadt, Germany) and injected on the gas chromatography/mass spectrometry and gas chromatography/tandem mass spectrometry (GC/MS/MS) system (consisting of a Varian Star 3400 CX GC system with a Chrompack CP-Sil 8 low bleed/MS column of $30 \mathrm{~m}$ and internal diameter of $0.25 \mathrm{~mm}$ with a film thickness of $0.25 \mu \mathrm{m})$. Pyrene and $\mathrm{BaP}$ were detected on a Saturn 4D, ion trap MS/MS (Thermo Varian, Palo Alto, USA), using the following ions: (i) $\mathrm{m} / \mathrm{z}$ of 202 for pyrene and (ii) $\mathrm{m} / \mathrm{z} 252$ and 253 for BaP. The limits of detection were 5.3 and $10.0 \mathrm{pg}$ for pyrene and $\mathrm{BaP}$, respectively. This resulted in limits of quantification for pyrene and $\mathrm{BaP}$ in the gas phase of $0.5 \mathrm{ng} / \mathrm{m}^{3}$ and $1.0 \mathrm{ng} / \mathrm{m}^{3}$, and in the particulate phase of 0.02 $\mathrm{ng} / \mathrm{m}^{3}$ and $0.04 \mathrm{ng} / \mathrm{m}^{3}$, respectively. The limits of quantification for the skin pads were $5 \mathrm{pg} / \mathrm{pad}$ for pyrene and $10 \mathrm{pg} / \mathrm{pad}$ for BaP. Pyrene (purity >99\%) and $\mathrm{BaP}$ (purity 98\%) were purchased from SigmaAldrich (Zwijndrecht, the Netherlands). The analysis was calibrated using standards prepared in toluene in the range 5.87-938 $\mathrm{ng} / \mathrm{l}$ for $\mathrm{BaP}$ and $1.48-237 \mathrm{ng} / \mathrm{l}$ for pyrene.

\section{Analysis from coal tar ointment}

After diluting CTO in tetrahydrofurane, we injected the sample directly into the chromatographic system without pre-treatment. The analysis was performed with an high performance liquid chromatography method with a gradient dilution and with a detection by fluorescence (excitation and emission wavelengths of 296 and $405 \mathrm{~nm}$, respectively, for $\mathrm{BaP}$ and 335 and $385 \mathrm{~nm}$, respectively, for pyrene).

\section{Urine analysis}

Urine was analyzed for both glucuronide-conjugated and free 1-OHP in glucuronidase/sulfatase hydrolyzed samples using the method of Jongeneelen et al (22) and urinary creatinine was analyzed using the Jaffé method. 1-OHP was analyzed on a high performance liquid chromatography system with a Lichrospher 100 RP-18 column with a particle size of $5 \mu \mathrm{m}$ (Merck, Darmstad, Germany) using fluorescence detection with a limit of detection of $2 \mu \mathrm{g} / 1$ and an interday repeatability of $12.6 \%$, based on an analysis of 24 samples in duplicate within a period of 12 months. Only the urine samples that were collected during study A were analyzed for 3-OHBaP. This analysis was performed according to the method described by Simon et al (23) with a limit of detection of $0.1 \mathrm{ng} / \mathrm{l}$, an intraday repeatability of less than $3 \%$, and an interday repeatability of less than $6 \%$.

\section{Calculations and statistical analysis}

The amounts of pyrene and $\mathrm{BaP}$ used in each patient treatment were calculated from the type and registered amount of CTO used. The concentration of 1-OHP in each urine sample was corrected by the urine creatinine content. Baseline excretion of 1-OHP was determined in the first void of urine after awakening, following a period of 48 hours not working with CTO.

The total excretion of 1-OHP was calculated from the recorded volumes for each void and the concentration was determined in an aliquot. In the analysis, no distinction was made between female or male nurses or smokers and non-smokers. Smoking is estimated to be of limited influence due to the study design (ie, comparison of urinary PAH metabolite excretion within each subject over time). Statistical testing was performed using SPSS version 14.0 (SPSS Inc, Chicago, IL, USA) using a paired Student's t-test for the results of study C. An unpaired Student's t-test was used if different nurses were compared before and after intervention (studies $A$ and $D)$. For comparison of the urinary excretion of 1-OHP in study A with the results of study D, only the results of urine samples collected during the first 24 hours were used.

\section{Results}

\section{Study A}

The levels of airborne pyrene and $\mathrm{BaP}$ were all below the limits of quantification, suggesting that inhalation exposure of pyrene and $\mathrm{BaP}$ due to the application of CTO is very unlikely. Therefore, inhalation of airborne pyrene and $\mathrm{BaP}$ can be ruled out as a source of occupational exposure.

During this study, all the nurses wore loose-fitting polyethylene gloves during at least part of each CTO treatment. Despite this good work practice, after one working shift substantial amounts of pyrene and $\mathrm{BaP}$ were observed on the nurse's hands. The distribution of the contamination of pyrene and $\mathrm{BaP}$ over three different pads on the same hand shows considerable inter-individual differences, indicating variations in the $\mathrm{PAH}$ content of different CTO products used and possible differences in working methods.

Table 2 presents the results of pyrene skin contamination testing and urinary excretion of 1-OHP. For nurses $\mathrm{A}$ and $\mathrm{K}$, the amount of CTO used is the same as they were treating the same patient. This is also the case for nurses $H$ and J. Nurses F and G were involved in the treatment of two patients resulting in the highest amount of administered CTO. However, 


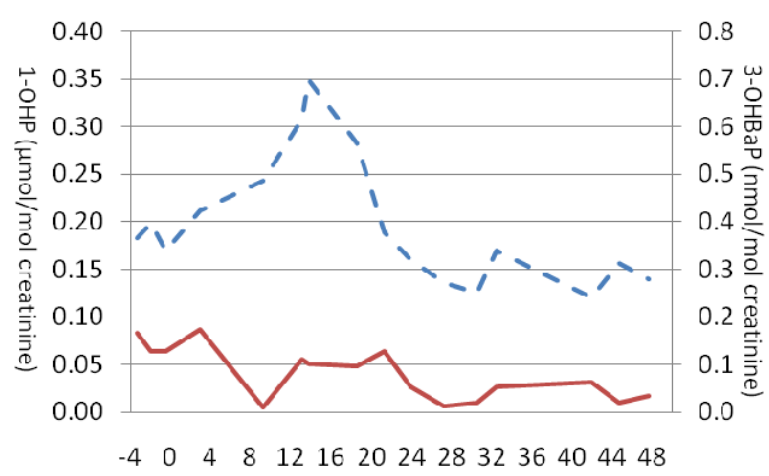

Figure 1. Pattern of excretion of 1-OHP (dotted line) and 3-OHBaP (straight line) in a male nurse (person $\mathrm{A}$ ) who reported smoking 1120 cigarettes/day and who treated one patient at 09:47 (time in hours; treatment started at $\mathrm{t}=0$ ). During the treatment, $55.8 \mathrm{~g}$ of dark CTO was applied during 20 minutes of working without gloves.

these two nurses did not have the highest skin contamination values or the highest excretion levels of 1-OHP in urine.

Pyrene applied as part of the CTO varies widely due to the use of different types of CTO with PAH contents ranging over three orders of magnitude. Similarly, the amount of $\mathrm{BaP}$ applied ranged from 1.7-25 $200 \mu \mathrm{g}$, with a median of $2758 \mu \mathrm{g}$ (not presented).

The highest level of excretion of 1-OHP was found in nurse A. The urine samples of this nurse were the only samples in which levels of $3-\mathrm{OHBaP}$ were detected (figure 1). Considering the amount of pyrene applied and the contamination of skin pads, this is certainly not the person with the highest external exposure to pyrene. The slight downward trend in both 1-OHP and $3-\mathrm{OHBaP}$ excretion patterns may be related to the use of CTO in treatment of a patient on the day before urine collection. Nurse A reported this exposure (not in line with the instructions) in the questionnaire. Since nurse $\mathrm{A}$ is also an active smoker, inhalation of tobacco smoke may have contributed to the excretion of $1-\mathrm{OHP}$ and $3-\mathrm{OHBaP}$.

The individual with the lowest urinary excretion of 1-OHP (nurse $\mathrm{K}$ ) was treating the same patient as nurse $A$, and had lower levels of contamination on the skin (except for contamination of the finger of $118 \mathrm{ng} / \mathrm{cm}^{2}$ ). It is likely that these two nurses divided the tasks during treatment of the patient in such a way that nurse A had more direct contact with CTO than nurse $\mathrm{K}$.

We also investigated the contamination of skin pads with $\mathrm{BaP}$. The levels observed on the pads were (median and range): (i) wrist: $6.5 \mathrm{ng} / \mathrm{cm}^{2}(<0.01-$ 105.1), (ii) palm: $3.9 \mathrm{ng} / \mathrm{cm}^{2}$ (1.2-79.7), and (iii) finger: $23.3 \mathrm{ng} / \mathrm{cm}^{2}(<0.01-98.0)$. The pyrene and $\mathrm{BaP}$ levels on the wrist and palm were highly correlated $\left(\mathrm{R}^{2}=0.95\right.$ and 0.97 , respectively). The correlation between pyrene and $\mathrm{BaP}$ on the finger pad was weaker $\left(\mathrm{R}^{2}=0.43\right)$ but still statistically significant $(\mathrm{P}<0.05)$.

We did not find statistically significant correlations between the urinary excretion of 1-OHP and work characteristics such as the total amount of CTO applied, the contamination of PYR on the skin pads,

Table 2. Amount of pyrene (PYR) used in coal tar ointment (CTO), skin contamination and excretion of 1-hydroxypyrene (1-OHP) during a period of 48 hours following the administration of CTO. (ND = not detected)

\begin{tabular}{|c|c|c|c|c|c|c|c|c|c|c|c|c|c|c|}
\hline \multirow{2}{*}{$\begin{array}{l}\text { Person } \\
\text { code }\end{array}$} & \multirow{2}{*}{$\begin{array}{c}\text { Age } \\
\text { (years) }\end{array}$} & \multirow{2}{*}{$\begin{array}{l}\text { Smoking } \\
\text { status }\end{array}$} & \multirow{2}{*}{$\begin{array}{c}\text { Number } \\
\text { of } \\
\text { treat- } \\
\text { ments }\end{array}$} & \multirow{2}{*}{$\begin{array}{c}\text { Total } \\
\text { treat- } \\
\text { ment } \\
\text { time } \\
\text { (min- } \\
\text { utes) }\end{array}$} & \multirow{2}{*}{$\begin{array}{c}\text { PYR } \\
\text { in CTO } \\
\text { applied } \\
(\mu \mathrm{g})\end{array}$} & \multicolumn{5}{|c|}{ Skin exposure to PYR } & \multicolumn{4}{|c|}{ Excretion of 1-OHP } \\
\hline & & & & & & $\begin{array}{c}\text { Wrist } \\
\left(\mathrm{ng} / \mathrm{cm}^{2}\right)\end{array}$ & $\underset{\left(\mathrm{ng} / \mathrm{cm}^{2}\right)}{\text { Palm }}$ & $\begin{array}{c}\text { Finger } \\
\left(\mathrm{ng} / \mathrm{cm}^{2}\right)\end{array}$ & $\begin{array}{l}\text { Mean } \\
\left(\mathrm{ng} / \mathrm{cm}^{2}\right)\end{array}$ & $\begin{array}{l}\text { Total } \\
(\mathrm{ng})\end{array}$ & $\begin{array}{c}\text { Total } \\
\text { excretion } \\
\text { (nmol/ } \\
48 \text { hours) }\end{array}$ & $\begin{array}{c}\text { Baseline } \\
\text { excretion } \\
\text { level a } \\
(\mu \mathrm{mol} / \mathrm{mol})\end{array}$ & $\begin{array}{c}\text { Highest } \\
\text { excretion } \\
\text { level } \\
(\mu \mathrm{mol} / \mathrm{mol})\end{array}$ & $\begin{array}{c}\text { Increase } \\
\text { from } \\
\text { baseline } \\
(\mu \mathrm{mol} / \mathrm{mol})\end{array}$ \\
\hline A & 51 & Smoker & 1 & 20 & 112 & 17.5 & 6.85 & 112 & 45.6 & 254 & 7.7 & 0.21 & 0.35 & 0.14 \\
\hline B & 39 & Non-smoker & 1 & 60 & 438 & 0.43 & 9.33 & 15.9 & 8.55 & 51.9 & 3.8 & 0.09 & 0.21 & 0.12 \\
\hline C & 28 & Non-smoker & 1 & 60 & 22000 & 173 & 15.6 & 189 & 126 & 762 & 1.5 & 0.01 & 0.18 & 0.18 \\
\hline $\mathrm{D}$ & 43 & Non-smoker & 1 & 20 & 6230 & 14.6 & 1.46 & 56.0 & 24.0 & 146 & 3.9 & 0.11 & 0.15 & 0.04 \\
\hline$E$ & 39 & Smoker & 1 & 5 & 36.2 & 2.71 & 2.02 & 18.8 & 7.84 & 47.6 & 2.2 & 0.10 & 0.13 & 0.03 \\
\hline $\mathrm{F}$ & 34 & Smoker & 2 & 90 & 31500 & 43.9 & 3.12 & 52.1 & 33.0 & 201 & 4.0 & 0.07 & 0.13 & 0.06 \\
\hline G & 40 & Non-smoker & 2 & 70 & 445000 & 165 & 28.4 & 9.58 & 67.5 & 410 & 2.2 & 0.06 & 0.11 & 0.06 \\
\hline $\mathrm{H}$ & 50 & Non-smoker & 1 & 20 & 83.8 & 300 & 202 & 49.0 & 184 & 1110 & 1.7 & 0.08 & 0.10 & 0.03 \\
\hline 1 & 22 & Non-smoker & 1 & 30 & 4970 & 0.19 & 0.32 & 9.4 & 3.30 & 20.0 & 1.7 & 0.19 & 0.10 & -0.09 \\
\hline$J$ & 49 & Non-smoker & 1 & 20 & 83.8 & 68.0 & 19.1 & 55.2 & 47.4 & 287 & 2.4 & 0.09 & 0.09 & -0.01 \\
\hline K & 44 & Non-smoker & 1 & 20 & 112 & 0.25 & 1.6 & 118 & 40.1 & 67.9 & 1.2 & 0.03 & 0.04 & 0.01 \\
\hline L & 29 & Non-smoker & 1 & 35 & 455 & 0.34 & 0.17 & ND & 0.17 & 1.10 & 1.3 & 0.05 & 0.04 & -0.01 \\
\hline
\end{tabular}

a Baseline excretion was determined in the first void of urine after awakening following a period of 48 hours not working with CTO. 
the duration of the treatment, or time elapsed between CTO application and washing hands.

\section{Study B}

The results from the three-week follow-up study are presented in figure 2 . Nurse 1 reported working a total accumulated period of 202 minutes without wearing gloves. Dark CTO was applied for only 22 minutes in total (on days 2, 3, 16, 17, and 19) while light CTO was used for the remaining 180 minutes (days 2, 3, 9, 12, 16,17 , and 19). Of the time used to apply CTO, $92 \%$ was spent without protection. During a shift where a significant amount of time was spent working unprotected (>30 minutes per shift), the urinary excretion of 1-OHP increased (days 3 and 17). However, on other days with a similar duration of working unprotected, no increase in urinary excretion of 1-OHP was observed (days 2, 9, 16, and 19). On day 12, working 10 minutes with unprotected hands resulted in a decrease of the urinary excretion of 1-OHP. The elevated level of excretion in the pre-shift sample on day 19 may be the result of a continued excretion of a relatively high skin absorption of pyrene on day 17.

For nurse 2, the reported duration of working with unprotected hands amounted to 93 minutes (15 minutes of which were spent using dark CTO and 78 minutes using light ointment). This nurse worked unprotected during $26 \%$ of all the time engaged in CTO application. Urinary excretion did not appear to be influenced by the application of СТO.

Nurse 3 reported the lowest total duration of work spent applying CTO (45 minutes) and only light CTO was used during this time. This nurse worked unprotected during $38 \%$ of the time spent applying CTO. The urinary excretion of 1-OHP also showed hardly any significant rise.

The total number of hours spent in unprotected direct handling of CTO led to higher levels of urinary excretion in nurse 1 who worked most of the time (and also a high percentage of "CTO application time") without gloves. However, this increase did not occur on all days where significant time was spent handling CTO. Use of (primarily) light CTO by nurses 2 and 3 did not result in a consistent rise in urinary excretion of 1-OHP.

\section{Study C}

Since studies A and B did not show a consistent elevated excretion of 1-OHP following skin exposure to CTO, a third study was conducted to find out if protecting hands with gloves would lead to a statistically significant lower uptake of PAH in a large group of nurses. Wearing loose-fitting polyethylene transparent gloves (King
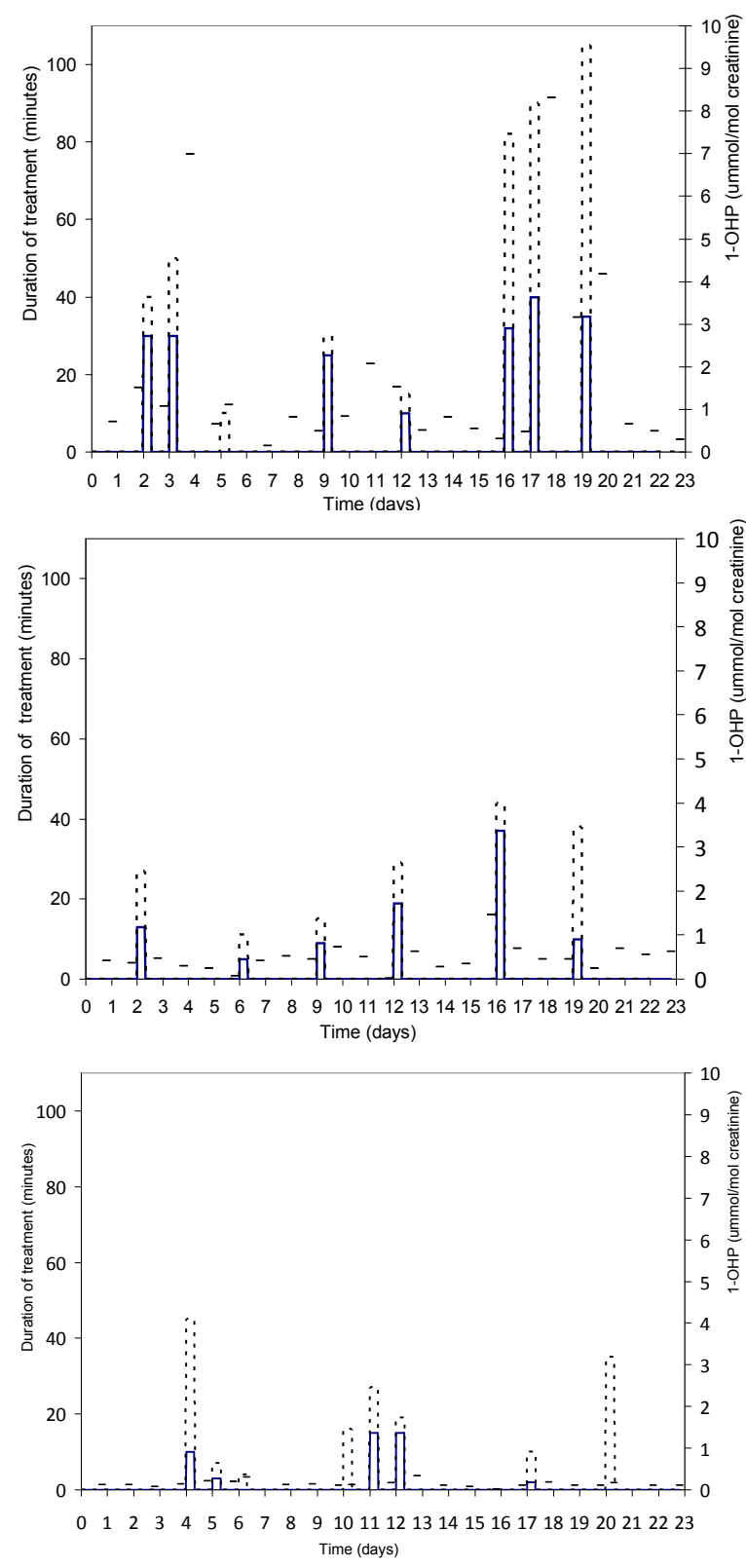

Figure 2. Duration of CTO treatment with gloves (dotted line) and without gloves (solid line) and excretion of 1-OHP in a post-shift (evening) urine sample (dashes). Results of three different nurses (non-smokers) are presented: nurse 1 upper panel, nurse 2 middle panel, and nurse 3 lower panel.

Gloves, Braintree, MA, United States), 35 nurses were asked to treat a single patient and to repeat the treatment in the same patient without the gloves. Repeating the topical application of CTO is part of the normal procedure in the clinical practice.

The results of the paired observations are presented in table 3. The wearing of gloves during a single treatment resulted in an average reduction of $51.5 \%$ in the total excretion of 1-OHP. The highest level of 1-OHP in a single spot urine sample decreased by $61.2 \%$. As 
Table 3. Median (range) or urinary excretion of 1-OHP in nurses working with and without gloves (study C).

\begin{tabular}{|c|c|c|c|c|c|c|c|c|}
\hline \multirow[t]{3}{*}{ CTO application } & \multicolumn{8}{|c|}{ Excretion of 1-OHP } \\
\hline & \multicolumn{2}{|c|}{$\begin{array}{l}\text { Total amount excreted } \\
(\mu \mathrm{mol} / 24 \mathrm{~h})\end{array}$} & \multicolumn{2}{|c|}{$\begin{array}{l}\text { Highest post-exposure level } \\
(\mu \mathrm{mol} / \mathrm{mol} \text { creatinine })\end{array}$} & \multicolumn{2}{|c|}{$\begin{array}{c}\text { Baseline } \\
\text { ( } \mu \mathrm{mol} / \mathrm{mol} \text { creatinine) }\end{array}$} & \multicolumn{2}{|c|}{$\begin{array}{l}\text { Change from baseline } \\
\text { ( } \mu \mathrm{mol} / \mathrm{mol} \text { creatinine })\end{array}$} \\
\hline & Median & Range & Median & Range & Median & Range & Median & Range \\
\hline Without gloves & 1.34 & $0.19-5.6$ & 0.31 & $0.06-0.98$ & 0.05 & $0.01-0.28$ & 0.23 & $0.00-0.74$ \\
\hline With gloves & 0.69 & $0.17-3.3$ & 0.12 & $0.04-0.70$ & 0.04 & $0.01-0.13$ & 0.06 & $0.00-0.57$ \\
\hline Change & -0.58 & $-5.1-1.0^{a}$ & -0.18 & $-0.81-0.04$ & 0.00 & $-0.14-0.09$ & -0.16 & $-0.74-0.02$ \\
\hline
\end{tabular}

a $P<0.001$

Table 4. Contamination of skin pads before and after introduction of a protocol for use of gloves and sleeves (studies $A$ and D). (PYR = pyrene, BaP = benzo[a]pyrene, ND = not detected $)$

\begin{tabular}{|c|c|c|c|c|}
\hline \multirow[t]{2}{*}{ Subgroup } & \multicolumn{2}{|c|}{$\begin{array}{c}\text { PAH contamination } \\
\text { before introduction of } \\
\text { skin protection protocol } \\
\text { (ng/pad) }\end{array}$} & \multicolumn{2}{|c|}{$\begin{array}{l}\text { PAH contamination } \\
\text { after introduction of } \\
\text { skin protection protocol } \\
\text { (ng/pad) }\end{array}$} \\
\hline & Median & Range & Median & Range \\
\hline \multicolumn{5}{|c|}{ Same nurses (paired observations, $\mathrm{N}=6$ ) } \\
\hline $\begin{array}{l}\text { PYR } \\
\text { BaP }\end{array}$ & $\begin{array}{l}349 \\
184\end{array}$ & $\begin{array}{l}146-1110 \\
60.5-394\end{array}$ & $\begin{array}{l}0.9^{a} \\
4.6^{b}\end{array}$ & $\begin{array}{l}\text { ND-35.6 } \\
0.2-31.7\end{array}$ \\
\hline \multicolumn{5}{|c|}{ Different nurses (unpaired observations, $\mathrm{N}=10-12$ ) } \\
\hline $\begin{array}{l}\text { PYR } \\
\text { BaP }\end{array}$ & $\begin{array}{l}173 \\
107\end{array}$ & $\begin{array}{c}1.1-1110 \\
5.5-394\end{array}$ & $\begin{array}{l}3.7^{b} \\
3.2^{b}\end{array}$ & $\begin{array}{c}\text { ND-93.5 } \\
0.5-171\end{array}$ \\
\hline
\end{tabular}

a $P<0.001$.

b $P<0.005$.

expected, the urinary excretion of 1-OHP at baseline (as observed from the first voids after awakening) was not different when working with or without gloves. The change from baseline to the maximum excretion of 1-OHP within 10 hours after CTO application was $74 \%$ lower when using gloves compared with working unprotected, but this result was not statistically significant.

\section{Study D}

A new work practice was introduced based on the use of latex-free polyvinyl chloride gloves and $50 \mathrm{~cm}$ Tyvek $^{\circledR}$ narrow sleeves with elastic on the wrist. With this new set of skin protection equipment, study A was repeated to verify if the reduction in uptake shown in study $\mathrm{C}$ would be feasible under more realistic exposure conditions. Since only six nurses were still working in the department, a paired analysis was performed in this subgroup. In addition, an unpaired statistical analysis was performed involving ten nurses from study A and 12 other individuals from study D. In both subgroups,
Table 5. Excretion of 1-hydroxypyrene (1-OHP) before and after introduction of a new protocol for the use of gloves and sleeves.

\begin{tabular}{|c|c|c|c|c|}
\hline \multirow[t]{2}{*}{ Subgroup } & \multicolumn{2}{|c|}{$\begin{array}{l}\text { Before introduction } \\
\text { of skin protection } \\
\text { protocol }\end{array}$} & \multicolumn{2}{|c|}{$\begin{array}{l}\text { After introduction } \\
\text { of skin protection } \\
\text { protocol }\end{array}$} \\
\hline & Median & Range & Median & Range \\
\hline \multicolumn{5}{|l|}{ Paired observations $(\mathrm{N}=6)$} \\
\hline $\begin{array}{l}\text { Total urinary excretion of } \\
1-0 \mathrm{HP} \text { (nmol in } 24 \text { hours) } \\
\text { Increase of urinary excretion } \\
\text { of } 1-\mathrm{OHP} \text { from baseline } \\
(\mu \mathrm{mol} / \mathrm{mol} \text { creatinine })\end{array}$ & 1.6 & $0.84-5.0$ & $0.69^{a}$ & $0.45-3.4$ \\
\hline \multicolumn{5}{|l|}{ Unpaired observations $(\mathrm{N}=10-12)$} \\
\hline $\begin{array}{l}\text { Total urinary excretion of } \\
1-0 \mathrm{HP} \text { (nmol in } 24 \text { hours) } \\
\text { Increase of urinary excretion } \\
\text { of } 1-0 \mathrm{HP} \text { from baseline } \\
(\mu \mathrm{mol} / \mathrm{mol} \text { creatinine })\end{array}$ & 1.5 & $-0.09-0.18$ & $0.64^{b}$ & $0.15-2.8$ \\
\hline
\end{tabular}

a $P<0.005$.

${ }^{b} \mathrm{P}<0.05$.

a substantial and statistically significant reduction of skin contamination of more than $97 \%$ was observed for both pyrene and $\mathrm{BaP}$ (table 4). The total urinary excretion of 1 -OHP was reduced by $56.9 \%$ in the paired analysis $(\mathrm{P}<0.05)$ and $57.3 \%$ in the unpaired analysis $(\mathrm{P}<0.05)$. The increase from baseline to the highest recorded value within 24 hours after the CTO showed a similar trend but no statistical significant reduction was observed (table 5).

\section{Discussion}

Pyrene and $\mathrm{BaP}$ were not detected in the gas phase and particle phase. For BaP, the air concentration was apparently lower that the previously reported background value for $\mathrm{BaP}$ in the particulate phase of outdoor air in the Netherlands of $0.05-0.15 \mathrm{ng} / \mathrm{m}^{3}$ (24). For the gas phase, our method was less sensitive (due to the lower air flow used during sample collection) but it is unlikely 
that $\mathrm{BaP}$ would be present in the gas phase when this substance was not observed in the particulate phase.

In contrast, significant amounts of pyrene and $\mathrm{BaP}$ were observed on skin pads attached to the dominant hand. The (calculated) amounts of $\mathrm{BaP}$ and pyrene applied to each patient using the different types of CTO showed a high correlation $\left(\mathrm{R}^{2}=0.97\right)$. On skin pads, correlations were low for the finger but higher for the wrist and the palm of the hand. This suggests that pyrene is useful as a chemical marker of $\mathrm{PAH}$ exposure while 1-OHP is a suitable biomarker of internal exposure to $\mathrm{BaP}$ in the case of CTO applications by nurses. This is important in this study because we were unable to detect urinary excretion of $3-\mathrm{OHBaP}$ in all but one nurse in study A.

No significant correlation was observed between the contamination of pyrene on the skin pads and the total amount of 1-OHP excreted in urine $\left(\mathrm{R}^{2}=0.11\right)$. This may be due to inter-individual differences in metabolism or skin absorption but also to the limited capability of estimating total skin exposure using skin pads. The skin contamination with CTO was not diffuse but showed some patchiness. This heterogeneity in skin contamination is difficult to assess when using skin pads.

Study B showed that working without gloves is associated with increased urinary excretion of 1-OHP in particular when using dark CTO (eg, nurse 1 in figure 2). This pattern is less pronounced in nurses 2 and 3 who worked fewer hours unprotected and used smaller amounts of dark CTO. A limitation to this finding is that the urinary excretion of 1-OHP is indicated by only one single post-shift urine sample per day.

In study $\mathrm{C}$, working with gloves showed that the group median 1-OHP excretion was 0.12 and the P95 value was $0.30 \mu \mathrm{mol} / \mathrm{mol}$ creatinine. When the nurses did not wear gloves, the median and P95 were 0.31 and $0.83 \mu \mathrm{mol} / \mathrm{mol}$ creatinine, respectively. The P95 values of 0.76 and $0.24 \mu \mathrm{mol} / \mathrm{mol}$ creatinine proposed by Jongeneelen et al $(9,25)$ were used to classify respectively smoking and non-smoking nurses as being occupationally exposed to PAH. In study C, 17 out of 35 workers would be classified as "occupationally exposed" when not wearing gloves, whereas only 2 workers would be classified as being exposed when wearing protective gloves. Therefore, wearing gloves created a situation that would be very close to what would be considered as "no occupational exposure" for the group of 35 nurses.

It is also interesting to compare the level of observed exposure with known sources of non-occupational "background" exposure $(20,26)$. The group median uptake of 1-OHP of $2.2 \mathrm{nmol}$ in 24 hours as calculated from table 2 is approximately $21.6 \%$ of what is estimated to be taken in on a daily basis from the diet of non-smokers, or only $23.9 \%$ of the amount estimated for active smokers if a complete conversion of pyrene to 1-OHP is assumed (20).

In 11 of the 12 nurses in Study A, there was no detection of 3-OHBaP. In part, this may be due to the method of collection and storage of urine leading to loss of 3-OH-BaP glucuronide. In one nurse, 3-OHBaP was detected in collected urine samples. This subject smoked $0.5-1$ pack of cigarettes per day. The urinary excretion for 3-OHBaP in nurse A (figure 1) was somewhat higher than the level that is normally seen in smokers (26), but well below the biological limit value of $0.45 \mathrm{nmol} / \mathrm{mol}$ creatinine proposed by Lafontaine et al (10). The highest value at 4 hours prior to the application of CTO and the gradually decreasing pattern of values suggests an uptake of $\mathrm{BaP}$ on the previous day. The latter was confirmed by a report from nurse $\mathrm{A}$ who acknowledged that she had treated a patient with CTO on that day. Moreover, excretion of 1-OHP showed a pattern indicating a possible work-related uptake of pyrene. This is consistent with a substantial skin contamination as indicated by a total $254 \mathrm{ng}$ of pyrene on all pads. The latency time between the moment of application (09:47 hours) and the highest value of urinary excretion (approximately 23:32 hours), suggests a delay of 13:45 hours, indicating skin absorption as the primary route of uptake rather than smoking (27).

The results of study B showed that especially the use of dark CTO when wearing no gloves might contribute to an uptake of PAH, as indicated by the increase of urinary excretion of 1-OHP from pre- to post-shift. These results indicated that the use of light CTO did not (in most cases) lead to such an increase and contributed to the explanation of the results observed in study $\mathrm{A}$, which showed that an increase in uptake of $\mathrm{PAH}$ is not observed in all subjects. Furthermore, these findings suggested that the role of skin protection should be further investigated.

The results of study $\mathrm{C}$ were related to a task-based study of exposure rather than a study of a real life work shift, which may involve the treatment of two or more patients during the course of a shift. The introduction of a new protocol for skin protection resulted in an almost complete elimination of skin exposure $(<3 \%$ residual exposure for pyrene and $\mathrm{BaP})$. In this work situation, skin absorption is considered to be the primary route of uptake since both inhalation and oral exposure are not very plausible routes of exposure. So it may be concluded that most of the occupational exposure to PAH due to the use of CTO was virtually eliminated. This major reduction in exposure did not lead to a complete reduction in the excretion of urinary metabolites due to the contribution of non-occupational sources of exposure to $\mathrm{PAH}$.

Study $\mathrm{C}$ was performed because it was still uncertain how effective a more stringent regime of skin protection 
would be in terms of reduction of PAH uptake. It was of particular interest to involve the nurses in this intervention study, comparing working with and without gloves under more or less standardized conditions. This participatory study design helped to convince the nurses of the added value of protecting their skin. The result of this study showed a substantial reduction that was also statistically significant based on the findings for the entire group. However, it should be made clear that, when looking at the results on an individual level, it appears the effectiveness may vary substantially from person to person: from a 5 -fold increase to a 15 -fold reduction.

In study $\mathrm{D}$, we showed that a twofold reduction of uptake of PAH (as indicated by excretion of 1-OHP) is feasible for a group of nurses working with CTO on a day-to-day basis inspite of the greater number of CTO treatments in study $\mathrm{D}$ (arithmetic mean 2.28, range 2-4) than in study A (arithmetic mean 1.17, range $1-2)$. It is obvious that this substantial reduction in the contamination of skin pads, would lead to a decrease in urinary excretion of 1-OHP, but the excretion observed was very close to levels seen in the general population at baseline values (9).

The influence of smoking on this result was evaluated by reanalyzing the data after excluding the smokers. The removal of the two smokers from the paired observations reduced the level of significance (the P-value changed from 0.007 to 0.022 ). The removal of three smokers from the unpaired observations caused the P-value to change from 0.037 to 0.214 , suggesting that interference of changes in smoking patterns on the excretion of 1-OHP cannot be excluded in this part of the analysis.

\section{Acknowledgements}

The authors would like to thank the nursing staff of the department of Dermatology at Radboud University Nijmegen Medical Centre who participated in this study. The authors also wish to acknowledge $\mathrm{T}$ de Boo for supervising the statistical analysis and $\mathrm{C}$ Klaver for her advice on the selection of personal protective equipment.

\section{References}

1. Arnold WP. Koolteer in de dermatologie: ook na het jaar 2000? [Coaltar in dermatology: also after the year 2000?]. Ned Tijdschr Gen. 1997;7:191-5.

2. Burton JL. "Health hazards" and the media. Lancet. 1996;347:200.
3. Plantin P. Faut-il interdire le coal tar? [Should we forbid coal tar?]. Ann Dermatol Venereol. 1997;124:205-7.

4. International Agency for Research on Cancer (IARC). Polynuclear aromatic compounds, part 4. bitumens, coal-tars and derived products, shale-oils and soots. Lyon: International Agency for Research on Cancer; 1985. IARC monographs on the evaluation of the carcinogenic risk of chemicals to humans, volume 35 .

5. Roelofzen JH, Aben KK, van der Valk PG, van Houtum JL, van de Kerkhof PC, Kiemeney LA. Coaltar in dermatology. J Dermatolog Treat. 2007;18:329-34.

6. Roelofzen JH, Aben KK, Khawar AJ, Van de Kerkhof PC, Kiemeney LA, Van Der Valk PG. Treatment policy for psoriasis and eczema: a survey among dermatologists in the Netherlands and Belgian Flanders. Eur J Dermatol. 2007;17:416-21.

7. Hansen AM, Poulsen OM, Menne T. Longitudinal study of metabolites of polycyclic aromatic hydrocarbons in urine from two psoriatic patients. Acta Derm Venereol. 1993;73:188-9.

8. Conflero E, Zordan M, Vneier P, Paleologo M, Levis AG, Cottica D, et al. Biological monitoring of human exposure to coal tar urinary excretion of total polycyclic aromatic hydrocarbons, 1-hydroxypyrene and mutagens in psoriatic patients. Int Arch Occup Environ Health. 1989;61:363-8.

9. Jongeneelen FJ. Benchmark guideline for urinary 1-hydroxypyrene as biomarker of occupational exposure to polycyclic aromatic hydrocarbons. Ann Occup Hyg. 2001;45:3-13.

10. Lafontaine M, Gendre C, Delsaut P, Simon P. Urinary 3-hydroxybenzo[a]pyrene as a biomarker of exposure to polcycyclic aromatic hydrocarbons: an approach for determining a biological limit value. Polycycl Aromat Compound. 2004:24:441-450.

11. Constantino JP, Redmond CK, Bearden A. Occupationally related cancer risk among coke oven workers: 30 years of follow up. J Occup Environ Med. 1995;37:597-604.

12. Evanoff BA, Gustavsson P, Hogstedt C. Mortality and incidence of cancer in a cohort of Swedish chimney sweeps: an extended follow up study. Br J Ind Med. 1993;50:450-9.

13. Hansen ES. Cancer mortality among Danish molders. Am J Ind Med. 1991;20:401-9.

14. Karlehagen S, Andersen A, Ohlson CG. Cancer incidence among creosote-exposed workers. Scand J Work Environ Health. 1992;18:26-9.

15. Partanen T, Boffetta P. Cancer risk in asphalt workers and roofers: review and meta-analysis of epidemiological studies. Am J Ind Med. 1994;26:721-40.

16. Ronneberg A, Andersen A, Mortality and cancer morbidity in workers from an aluminium smelter with prebaked carbon anodes, part II. Occup Environ Med. 1995;52:250-4.

17. Scotto J, Fraumeni JF. Skin (other than melanoma). In: Schottenfeld D, Fraumeni JF jr, editors. Cancer epidemiology and prevention. Philadelphia (PA): Saunders; 1982. p 996-1011.

18. Tremblay C, Armstrong B, Thériault G, Brodeur J. Estimation of risk of developing bladder cancer among workers exposed to coal tar pitch volatiles in the primary aluminum industry. Am J Ind Med. 1995;27:335-48. 
19. Buckley TJ, Lioy PJ. An examination of the time course from human dietary exposure to polycyclic aromatic hydrocarbons to urinary elimination of 1-hydroxypyrene. Br J Ind Med. 1992;49:113-24.

20. VanRooij JGM, VanLieshout EMA, Bodelier-Bade MM, Jongeneelen FJ. Effect of the reduction of skin contamination on the internal dose of creosote workers exposed to polycyclic aromatic hydrocarbons. Scand J Work Environ Health. 1993:19:200-7.

21. Jongeneelen, FJ, Scheepers PTJ, Groenendijk A, Van Aerts LAGJM, Anzion RBM, Bos RP, et al. Airborne concentrations, skin contaminations and urinary metabolite excretion of polycyclic aromatic hydrocarbons among paving workers exposed to coal tar derived from road tars. Am Ind Hyg Assoc J. 1988;49:600-7.

22. Jongeneelen FJ, Anzion RBM, Henderson PT. Determination of hydroxylated metabolites of polycyclic aromatic hydrocarbons in urine. J Chromatogr. 1987;23:227-32.

23. Simon P, Lafontaine M, Delsaut P, Morele Y, Nicot T. Trace determination of urinary 3-hydroxybenzo[a]pyrene by automated column-switching high-performance liquid chromatography. J Chromatogr B. 2000;748:337-48.

24. Buijsman E. Assessment of air quality for polycyclic aromatic hydrocarbons in the Netherlands. Bilthoven (Netherlands): National Institute of Public Health; 1999. Report number 729999001.

25. Jongeneelen FJ. Biological exposure limit for occupational exposure to coal tar pitch volatiles at coke ovens. Int Arch Occup Environ Health. 1992;63:511-6.

26. Lafontaine M, Champmartin C, Simon P, Delsaut P, FunckBrentano C. 3-Hydroxybenzo[a]pyrene in the urine of smokers and non-smokers. Toxicol Lett. 2006;162:181-5.

27. Viau C, Vyskočil A. Patterns of 1-hydroxypyrene excretion in volunteers exposed to pyrene by the dermal route. Sci Total Environ. 1995;163:187-90.

Received for publication: 30 June 2008 